\title{
Alien Intruders in Relevant ARIThMETIC
}

\author{
Robert K. Meyer and Chris Mortensen \\ Australian National University \\ University of Adelaide
}

The system $R^{\sharp}$ of first-order relevant arithmetic was introduced in [12], as the result of adding the (first-order version of the) Peano postulates to relevant predicate calculus RQ. The following model was exhibited to show the system non-trivial (thus partially circumventing Gödel's Second Theorem). We pick as our domain $\mathbf{D}$ of objects the integers $\bmod 2$, with $+, \cdot, 0$ interpreted in the obvious way; on this plan, the successor operation ' is evidently interpreted so that $0^{\prime}=1$ and $1^{\prime}=0$. As our collection $\mathbf{V}$ of truth-values we pick the set $\mathbf{3}=\{\mathrm{T}, \mathrm{N}, \mathrm{F}\}$, with sentential connective $\&, \vee, \sim, \rightarrow$ defined on the (classical) subset $\mathbf{2}=\{\mathrm{T}, \mathrm{F}\}$ in the usual classical way. To complete the definition of connectives on $\mathbf{3}$, we define

\begin{tabular}{|c|c|c|c|c|c|c|c|c|c|c|c|c|c|}
\hline$\&$ & $\mathrm{~T}$ & $\mathrm{~N}$ & $F$ & V & $\mathrm{T}$ & $\mathrm{N}$ & $F$ & $\rightarrow$ & $\mathrm{T}$ & $\mathrm{N}$ & $F$ & & $\sim$ \\
\hline $\mathrm{T}$ & $\mathrm{T}$ & $\mathrm{N}$ & $F$ & $\mathrm{~T}$ & $\mathrm{~T}$ & $\mathrm{~T}$ & $\mathrm{~T}$ & $\mathrm{~T}$ & $\mathrm{~T}$ & $F$ & $F$ & $\mathrm{~T}$ & $F$ \\
\hline $\mathrm{N}$ & $\mathrm{N}$ & $\mathrm{N}$ & $F$ & $\mathrm{~N}$ & $\mathrm{~T}$ & $\mathrm{~N}$ & $\mathrm{~N}$ & $\mathrm{~N}$ & $\mathrm{~T}$ & $\mathrm{~N}$ & $\mathrm{~F}$ & $\mathrm{~N}$ & $\mathrm{~N}$ \\
\hline$F$ & $\mathrm{~F}$ & $\mathrm{~F}$ & $\mathrm{~F}$ & $F$ & $\mathrm{~T}$ & $\mathrm{~N}$ & $\mathrm{~F}$ & $F$ & $\mathrm{~T}$ & $\mathrm{~T}$ & $\mathrm{~T}$ & $\mathrm{~F}$ & $\mathrm{~T}$ \\
\hline
\end{tabular}

People familiar with relevant logics will recognize $\mathbf{3}$ so characterized as the 3-point Sugihara matrix $\mathrm{S}_{3}$ (so-called, no doubt, because it was invented by Sobociński, and usefully introduced into the study of relevant logics by us and a number of other people, after which it has had a habit of appearing, sometimes in disguise, in most papers on the subject). People unfamiliar with relevant logics will recognize the truth-tables for $\&, \vee, \sim$ as those of Lukasiewicz's original 3-valued logic (the only intuitive way of settling these tables when $\mathrm{N}$ (euter) is taken as an intermediate value between $\mathrm{T}(\mathrm{rue})$ and 
$\mathrm{F}$ (alse), despite all the clashing proposals made since); while $\rightarrow$ departs from Eukasiewicz only on the prescriptions $\mathrm{N} \rightarrow \mathrm{N}=\mathrm{N}$ and $\mathrm{T} \rightarrow \mathrm{N}=\mathrm{N} \rightarrow \mathrm{F}=\mathrm{F}$. (Basically this means that Eukasiewicz was still under the influence of that classical lassitude which tends to assign statements higher truth-values than they deserve.)

It is now easy to say what our non-trivializing model $\mathbf{M}_{2}^{3}=\langle\mathbf{D}, \mathbf{V}\rangle$ for $\mathbf{R}^{\sharp}$ was. Modulo 2, there are only 4 atomic sentences whose truth-values we have to settle; we settle them by setting to $\mathrm{F}$ the values of $0=1$ and $1=0$, and to $\mathrm{N}$ the values of $1=1$ and $0=0$. Truth-functional combinations of these sentences are then assigned values by the 3-valued tables. And interpreting quantifiers is almost as easy. Modulo $2, \forall x A x$ is simply to be interpreted as $A 0 \& A 1$ (since 0 and 1 are all that there is, on the interpretation, to quantify over), with $\exists x A x$ interpreted dually. While we can't say that all the theorems of $R^{\sharp}$ are true in this model (after all, we started $0=0$, which looks pretty true, off at the intermediate truth-value $\mathrm{N}$ ), we can do almost as well. All the axioms of $\mathrm{R}^{\sharp}$ are never false (i.e., never take the truth-value $\mathrm{F}$ in the model), and this property is preserved under the relevant logical rules (modus ponens for $\rightarrow$ and adjunction for \&). (Since the reader may expect some trouble to come up over induction, let us briefly ease its mind on the point. (We assume that this paper will be published in machine-readable form.) Induction tells us that whatever holds of 0 and all its successors holds of all numbers. In this model, what holds of 0 and all its successors holds of 0 and 1, and hence of "everything." End of verification.) But $0=1$ was assigned $\mathrm{F}$ from the start. It is false, even $\bmod 2$. so, since we can only prove in $\mathrm{R}^{\sharp}$ things that are never false in $\mathbf{M}_{2}^{3}$, we can by no means prove that $0=1$. And the argument (which the reader is invited to check in technical detail) is completely elementary. And so $R^{\sharp}$ is consistent. (In at least one standard sense of this overused term, and in fact in several.)

How did Gödel, who was reputedly pretty sharp, miss this? For all the metaphysical garbage that his work has called forth, spinning out hierarchies of ever more dubious systems for the purpose of demonstrating the nontriviality of the last dubious system, anything which starts counting at 0 and which can get as far as 1 is perfectly adequate to dismiss $0=1$ and related nonsense. As all of us would have thought, until we were informed that there were deep theorems of mathematical logic to the contrary. (So if any 8-bit machines - or, for that matter, 1-bit machines - are reading this paper, take heart. For all the talk that you are obsolete, even you can do what Gödel is reputed to have claimed you couldn't.)

Australasian Journal of Logic (18:5) 2021, Article no. 7 
But, having dwelt elsewhere at some length on Gödel's Oversight (specifically, in [10], which should sometime see the light of day), we shall be brief about it here. Gödel erred in taking the word of Whitehead and Russell (and manufacturers of related systems) that they had "reduced" arithmetic to logic. (Though "erred" is too strong here, since [7] already recognizes that systems which are different enough from the ones studied in [10] are not necessarily subject to its limitations. Or, in the case of relevant arithmetics, they remain subject to them, but in a way less destructive of mathematical certitude and less crippling to formal epistemology.) The classical logic that was the target of the reduction was a cut-price brand, which conflates many important ideas that are distinguished in ordinary mathematical and linguistic practice. (Cf. our remarks about overloading the word "consistent.") Basically, the steps in the argument connecting the unprovability of the Gödel sentence "17 Gen r" (which, however one looks at it, is a pretty queer sentence, in addition to being several miles long when put into primitive notation) to the unprovability of garden variety falsehoods like " $0=1$ " depend on fallacies of material implication. If, unimpressed by the classical proclivity to upgrade to "true" putative logical principles whose actual and intuitive truth-value is "false," we block some of these fallacies, falling into subtle confusions no longer produces garden variety falsehoods willy-nilly. Let us put it this way. What if " 17 Gen $r$ " were provable? We have, after all, no properly arithmetical grounds to rule it out, and must help ourselves to heaps of the standard set-theoretic mythology to sustain the faith. Were the mythology to crack and the faith to fail, what is the words thing that could happen to us? At first glance, it is that we shall have a proof of $\sim(17$ Gen $r$ ) as well. But, at second glance, we may recall that, at least according to some people, $A$ and $\sim A$ together imply what you please. If what you please is " $0=1$," we shall then have a proof of a garden variety and wholly unsubtle falsehood in one more step.

Now it is this sort of thing that we are against, no matter how often established logical authorities and their burgesses try to con us into the view that materially valid inferences are always O.K. Not here they aren't! Subtle contradictions do creep into people's reasoning. Nor is there any ultimate defence against the possibility that even humdrum formal systems in "safe" subjects like arithmetic have got caught in the confusion. Note that we said possibility. It is for future researchers to discover if we are actually confused. (Unfortunately, the only decisive answer that they can give us to that question is Yes.) For the philosophically exciting, epistemologically injurious, 
and ultimately most insane conclusion drawn from Gödel's Theorem is that we cannot, really, be very sure about anything mathematical. By natural extension, since simple arithmetical knowledge is generally account as secure knowledge, we cannot, really, be very sure about anything, at all.

This is a high philosophical price to pay for the technical success of some sneaky self-referential tricks. (They are, to be sure, impressive tricks.) For, in plain words, it simply does not follow from the fact that we are possibly confused about some things that we are possibly confused about everything. Truthfully, Hilbert's formalist optimism is in a way to blame here. Hilbert wished to make all of mathematics secure, all together, by demonstrating the consistency of a suitable Super System, using methods sufficiently elementary that everybody would have to accept them. ("Everybody" in those days included even the mathematical intuitionists, which was felt to show sufficient obeisance to Fogelin's Rule - "No funny business!" [1, p. 106].) This project failed. It's hindsight, but it deserved to fail. Who expects, or should have expected, an ironclad guarantee that mathematicians have hitherto been right about everything? But, fatuous optimism not having worked, there was all too much fatuous pessimism in the conclusions drawn. (These days - and most days, we suspect, among workaday mathematicians who take their subject as they find it - fatuous fideism is rather more in vogue. If the logicians failed in their efforts to put mathematics on a sound basis, some can do no better than to take Tennyson's advice, "Believing where we cannot prove." Even in the whole hierarchy of ZF sets, or more. But, while we have never wished to knock other people's religions, a more credible line needs to be drawn between the domain of Special Revelation and that of Reason in these things.) To show "17 Gen $r$ " unprovable, maybe we do have to be able to count to $\varepsilon_{0}$ (in whatever sense that is possible). But it is madness if we have to count to $\varepsilon_{0}$ to stave off a demonstration that $0=1$. For any child would laugh to see one. And if our formal systems cannot contain the contagion of subtle trickery into the domain of plain facts, even potentially, then it is time to send out for better systems. Fortunately, while not all of the technical evidence is yet in, we appear to have such better systems for arithmetic in $R^{\sharp}$ 
and related theories. ${ }^{1}$ Meanwhile, having to count to $\varepsilon_{0}$ every time we want to show some simple arithmetic falsehood undemonstrable is like sending a nuclear device to do the work of a flyswatter. What we can do by counting to 2 - or to $6,14,42$, or 3088 - let us by all means do. We shall spare ourselves ontological, epistemological, and other dangerous fallout.

While we have anticipated ourselves slightly, it is no less evident that we can construct mod-whatever-you-please models on the same plan that we used to construct our mod 2 model. (This is useful if one wants to show $2+2=2$ is likewise unprovable; or $\forall x \forall y(2 \cdot x=2 \cdot y)$, and so forth.) We get a variety of further, more general consistency results this way, removing not only primary school arithmetic but high school algebra from the range of the Gödelian fist. We also get a number of further models that are worthy objects of mathematical study in their own right. (See in addition to op. cit., [13] and [15] as well.) Dunn's [6] is an interesting related contribution.) All of this (and further Mortensen papers now in preparation or soon to appear, such as [16]) belongs to the topic of Inconsistent Model Theory. (Note that our initial model was intuitively inconsistent by making lots of sentences - for example, $0=0$ - never false by assigning both them and their negations the intermediate truth-value $\mathrm{N}$.)

We shall use the remainder of this note to make a modest addition to the topic of Inconsistent Model Theory, building both on what we have said above and our work elsewhere. (Though, unless the reader is insistent on checking axioms and the like, which we have left it to look up, this paper will be selfcontained. For the insistent, RQ was first formulated in [2]. The arithmetical postulates to add to get $R^{\sharp}$ are those set out in [4, p. 42]. (Caution: take these postulates exactly in the form found in [4]; do not substitute classical equivalents for them, which are not necessarily relevantly equivalent. And add $x=y \rightarrow x^{\prime}=y^{\prime}$ and $x=y \rightarrow(z=y \rightarrow x=z)$, which suffice with the other postulates to give identity its expected properties, again for the relevant context.) An essentially equivalent formulation of $R^{\sharp}$ appears in [12].)

\footnotetext{
${ }^{1}$ Burgess [3] argues - or rather pronounces - to the contrary, in remarks based apparently on its author having got access to our unpublished work. This raises some questions of propriety. Moreover, the actual account of $\mathrm{R}^{\sharp}$ given is a straightforward hatchet job, misunderstanding some things and misrepresenting the rest. Assign most of its assertions the truth-value F. Assign the rest N. Do not, on any account, assign T to anything in Burgess' paper that bears on the present subject, except perhaps for the author's institutional affiliation.
}

Australasian Journal of Logic (18:5) 2021, Article no. 7 
We have formulated $\mathrm{R}^{\sharp}$ be adding the first-order Peano postulates to a relevant quantificational base. But why do that? Even classically, these postulates are known to be seriously incomplete at this level. (To be sure, any level at which they are complete gets into what we have labelled "fatuous fideism.") In fact, there is an alternative $\mathrm{R}^{\sharp}$, which adds an $\omega$-rule to $\mathrm{R}^{\sharp}$ ("If for each numeral $n$ the theorem $A(n)$ has been demonstrated, infer $\forall x A(x)$ "), and which, taking the Standard Mythology for granted, contains exactly the standard arithmetical truths in the truth-functional part of its vocabulary. (Note that our model $\mathbf{M}_{2}^{3}$, and all models in the same vein, makes all theorems of $\mathrm{R}^{\sharp \sharp}$ never false as well. For it does not, after all, involve us that deeply in fatuous fideism if our domain of quantification is finite.) Moreover, we can of course switch the logical base. For $\mathbf{M}_{2}^{3}$ and its ilk satisfy not only $\mathrm{R}$ but the stronger system RM and its extensions, which give rise on the same plan to arithmetics $\mathrm{RM}^{\sharp}$, etc. (Most of our work in [13] dwelt directly with these stronger systems.)

Still, despite its deficiencies, first-order arithmetic seems like an interesting test case for application of the ideas of relevant logics. And we have seen that the imposition of relevant distinctions does make a difference, even if our interest in $R^{\sharp}$ is ultimately encompassed by interest in a stronger and more comprehensive system that contains it. And let us now turn to the specific postulates that first-order arithmetic has traditionally been required to satisfy. Taking the others as more or less straightforward and uncontroversial, we shall concentrate on the postulate of mathematical induction. It is, after all, by far the most complicated of the postulates; and, without second-order quantifiers, it boils down to infinitely many separate instances. Nonetheless, it is also the postulate to which fingers point when the deficiencies of first-order arithmetic are being bewailed. For while, on the usual extensional intuitions, we want to assert mathematical induction for uncountably many sets of natural numbers (although it can only be asserted usefully for just one such set - namely $\mathbb{N}$ itself, all other sets of natural numbers either failing to contain 0 or failing to contain the successor of some member), a countable language offers only countably many one-place open sentences to stand in for sets in our formulation of the induction postulate. So, unless we are terribly lucky (and we now know that we weren't), it is dubious that we have expressed enough of the induction postulate to characterize the natural numbers as definitively as he had hoped.

Such is a bit of the conventional wisdom (or at least of the conventional alibi for what went wrong). But what motivated people to pick mathematical 
induction as a postulate on the natural numbers in the first place? One reason, we cannot help but think, is that this principle and its close relatives are so enmeshed in the ordinary proof procedures applied in number theory that nobody ever thought seriously of not having it. But, at least according to Wang in [20], there was considerably more to it historically than that. One of the reasons, which is frequently pointed out, why mathematical induction is such a natural proof procedure is that it takes seriously how the natural numbers are constructed, being built up from 0 by adding 1's. From this viewpoint, the principle is nothing but the assertion that if a predicate holds of the number we start with, and if its holding is preserved when we construct each number from the last, then most certainly this predicate will hold of the whole lot.

But what if, despite our good intentions on the matter, some impostor manages to masquerade as a number, without having been got from 0 from adding 1's? That effective formal systems are unable to unmask such impostors is at once their shame and glory, motivating the title of this paper, and much else. but they have at least made the effort. The effort is called "mathematical induction," and its place among the arithmetical postulates is the chief product of the axiomatic approach to number theory initiated by Grassmann in [8] and given its modern form in the work of Dedekind (which, scholarship having been up to its usual standards, has caused us ever since to identify the governing principles of arithmetic as the Peano postulates).

But let us hear Dedekind himself on the subject of unmasking impostors ("Letter to Keferstein," translated in [19, p. 100]). "What then must we add... in order to cleanse our system $\mathbf{S}$ of such alien intruders as disturb every vestige of order and to restrict it to $\mathbb{N}$ ? This was one of the most difficult points of my analysis and its mastery required lengthy reflection."

It is a chilling prospect that, among the integers that God made, there might be smuggled in some little green numbers of other ancestry. Could it be that, when these integers stand up to be counted, there is an E.T. among them, which manages sufficiently to resemble its fellows that even the most distinguished (mathematical) scientists are deceived? Might it even be that there is a whole host of these invaders, whose cover is that they appear to satisfy the Dedekind-Peano postulates for the natural numbers but which no effective specification of these numbers can unmask as phonies?

Well, yes. These things can be. And are, despite the confident quotation just cited. The idea was that mathematical induction (or one of its equivalents) so constrained what was to count as a number, in a model of the 
postulates, that no little green numbers would get in. For the logistic idea had been to put elementary arithmetic on an indisputable basis by laying down postulates from which all and only the arithmetical truths follow. For a while, there was considerable confidence that this had been done successfully. Then Gödel struck, and things have never been the same again. (To be sure, the affinity of our century for wars, revolutions, recessions, famines, and the threat of atomic extinction might also depress some people. But we are speaking here of mathematical depression, which we take to be of a higher intellectual order.) Granted, the logistically faithful can still hold that Dedekind got it just right; and that, essentially, his postulates have only one model, which is the collection $\mathbb{N}$ of honest natural numbers. (Dedekind did have a categoricity proof, after all.)

But the original logistic idea was struck down by the inadequacy of the accompanying deductive apparatus, a point that we have been dwelling on above. The semantic reflection of this blow, dealt by Skolem in [17] (even before [7]) lies in the existence of non-standard models for $\mathbb{N}$ (and much else). On the Standard Mythology (that is, taking the "honest" natural numbers $0,1,2$, etc., for granted, where you are supposed to know what is meant by "etc."), these non-standard models differ from $\mathbb{N}$ exactly by admitting alien intruders - hordes and hordes of them, each of them "infinite" from the viewpoint of $\mathbb{N}$. (For each little green number is bigger than 0 , bigger than 1, und so weiter.) Then, for the faithful, the question becomes, "How do we formulate the Standard Mythology so that its non-numeric objects (for example, sets to whose existence we appeal in reconstructing Dedekind's categoricity proof for $\mathbb{N}$ ) are themselves shielded from perverse reinterpretation?" Answer - for pessimists - "It isn't possible." For the germ of the Dedekind-Peano idea is that $\mathbb{N}$ is the intersection of the successorclosed sets containing 0 . (That's what mathematical induction was doing, at least in intent.) If the Honest Natural Numbers are among the sets whose intersection is being taken, this set will surely be a subset of all the others; whence the idea does produce the standard $\mathbb{N}$. But what if the Honest Natural Numbers do not constitute a set at all, at least from the viewpoint of some particular model? Then the procedure does not secure its intended effect. The $\mathbb{N}$ produced is bogus, at least in the sense of containing alien intruders. Only given a preferred model of set theory (or other foundational apparatus) can we insist that $\mathbb{N}$ is, near enough, what we wanted it to be. And it is truly and not just formally perverse if, to believe the Standard Number-Theoretic Mythology (which does come naturally to most of us - or

Australasian Journal of Logic (18:5) 2021, Article no. 7 
seems to, since we were all drilled in it for years) we must believe first in some Set-Theoretic Mythology, or other Tall Tale, which has got to be more problematic, not less.

So, all in all, if one wants to Keep the Faith in these matters, the least committal course would seem to be simply to believe in $\mathbb{N}$, and be done iwth it. (For the remainder of this note, we shall at least talk as if we did. But the reader is to draw from that no further conclusions about our (mathematical) religion than we have drawn about its.) We shall take $\mathbb{N}$ as the standard model of arithmetic. (Everybody else does.) Other models (whatever formal theories they may be associated with, including set theories and classical and non-classical arithmetics) we call non-standard. The standard model of arithmetic being pretty boring - almost everybody thinks that he or she knows what it is supposed to be, and is only annoyed at not being able to characterize it - it is non-standard model theory that has boomed over the last decade or two. So, little green numbers being in style, let us see whether we can crowd more of them into a model of $\mathrm{R}^{\sharp}$ than the competition can offer.

Let us return to $\mathbf{M}_{2}^{3}$. It is simple enough, and constitutes a paradigm for the kinds of unexpected models that satisfy systems of relevant arithmetic. To put the reader's mind to rest, all the expected models are there also, both standard and non-standard. For there are two components of a relevant model; a domain of objects, and a domain of truth-values, both with operations appropriate to their category (e.g., + for objects, and \& for truthvalues) defined upon them. Nothing prevents us from taking the standard $\{\mathrm{T}, \mathrm{F}\}$ as our truth-value domain, with operations defined classically. If we do, the models for $R^{\sharp}$ under this restriction will be exactly the ones for the corresponding classical first-order Peano arithmetic P. So we do not lose any models.

But we do gain some. Indeed, it is pleasant to find the most natural models of the integers - the ones that one finds in the first few pages of any text on abstract algebra, namely the integers $\bmod n$ for finite $n$-among the models for $\mathrm{R}^{\sharp}$. For note that, whatever we do with truth-values, our collection of objects and associated operations in a model may be characterized independently as whatever sort of abstract algebra it is. For $\mathbf{M}_{2}^{3}$, this domain $\mathbf{D}$ is a very pleasant algebraic object indeed; for the integers mod 2 constitute a ring under the operations - and +; and, for that matter, a field. This may be an inconsistent model. But it is a nice one. And, while perhaps we should have learned by now to expect no better, one of the more annoying 
things about non-standard models of $\mathrm{P}$ is that they are not nice. (Still, there are those who have learned to love them.) Except that these models of $\mathrm{P}$ are collections of authentic numbers and little green numbers that have set up housekeeping together - ordering themselves on the aforementioned plan that all the authentic members of $\mathbb{N}$ come first (well, it's nice that they have some priority), in their natural order, with infinitely many copies of the integers following in slab after slab, the slabs themselves being as densely packed as if they were rational numbers. (The jargon is that these models have order type $\omega+\left({ }^{*} \omega+\omega\right) \cdot \eta$, which is not an order type that you would like to bring home to Mother, unless she has cooked an exceptionally large meal.)

Well, there is something perverse going on if the logician can countenance structures of the latter kind as modelling arithmetic, while ruling out the more natural and familiar mod models as models of the natural numbers. What's perverse about it? It is only by extensional courtesy (which the category theorists may be in the process of withdrawing) that we think of the natural numbers and their associated operations and relations as collections of objects at all. What they are, more fundamentally, are rule-governed structures. If, for whatever reason, we want tot think about models of a theory other than the intended one(s), it is the rules that go into the stipulation of the intended structure that we most want to preserve, in as simple, clear, and natural a way as possible. While we may get some other sorts of models anyway - just because our original stipulation was incomplete, ${ }^{2}$ and perhaps ineluctably so - they are in some sense accidental models.

There is another path, which as [6] points out is more familiar to the algebraist. Instead of getting extra models by underloading a theory-i.e., having a theory satisfy fewer constraints than we really want, because it is incomplete - we can also get interesting models by overloading that theoryintuitively, making it inconsistent by identifying things that, really, we take to be distinct. This is the effect of the algebraist's morphism, if we think about it. There is a function $h$ from the natural numbers to the integers mod 2 , which takes the even integers onto 0 and the odd ones onto 1 , preserving meanwhile the chief algebraic operations on integers. From the viewpoint of $\mathbb{N}$, this is a confused picture. $\mathbb{N}$ wants to distinguish 6 from 16, though they are not distinguished mod 2. But the picture is not a completely confused

\footnotetext{
${ }^{2}$ Negation-incompleteness, to be sure, is only part of the story, though it yields by the completeness theorem for 1 st-order logic that systems like $\mathrm{P}$ will have unintended models. But there is also the point that formal methods are incomplete. So, by Skolem, even negation-complete theories may have such models.
}

Australasian Journal of Logic (18:5) 2021, Article no. 7 
one. Indeed, since the computers that run the world these days do their own integer arithmetic mod 2-to-the-power-of-something, one can get a great deal done in this confused picture. And there is no reason in the world why a model that results from fruitful confusion (because it corresponds to intuitive inconsistency) is less interesting or worthy of study than one which results from fruitful ignorance (corresponding similarly to incompleteness).

So Dunn's view of these things in [6] is, we think, very nicely put. Moreover, being algebraically familiar, it belongs in the logician's bag of tricks. But there is a realm, to which we now turn, where we can make use of what goes with incompleteness and what goes with inconsistency together. It is the realm of inconsistent non-standard odels of arithmetic. For just as we have shown that the standard model $\mathbb{N}$ of classical Peano arithmetic $\mathrm{P}$ can be collapsed $\bmod n$, for finite $n$, to make the integers $\bmod n$ a model of $\mathrm{R}^{\sharp}$, just so we can collapse an arbitrary non-standard model $\mathbf{M}$ of $\mathrm{P} \bmod$ an "infinite integer" $n$ to make these "integers mod $n$ a model of the relevant Peano arithmetic $R^{\sharp} .{ }^{3}$ Note the interplay of inconsistency and incompleteness here. Because $\mathrm{P}$ is incomplete, it has non-standard models. But no homomorphic image of one of these models is a model of $\mathrm{P}$, because 0 becomes a successor. When eyeball-to-eyeball with inconsistency $P$ blinks. But $R^{\sharp}$, we shall see, does not blink. Its models preserve the distinctions that homomorphic images preserve, while still being models of all the first-order Peano postulates. Moreover, some of these inconsistent non-standard models are intrinsically quite interesting, as we shall see. Here's how to form them. (Now follows the part of the paper which readers more interested in impressions than technical details may wish to skip.)

Let $\mathbf{M}$ be an arbitrary model of $\mathbf{P}$. While $\mathbf{M}$ is not quite a ring, it is near enough that the usual methods of forming homomorphic images will work. We shall continue, by courtesy, to refer to the elements of $\mathbf{M}$ as "integers." (At least the ordinary natural numbers are among these integers, as we have seen, together with whatever alien intruders have sneaked in.) And we shall also continue to use " $\mathbf{M}$ " to refer indifferently to its base set; to the resulting structure in the algebraic sense, with appropriate operations $+, \cdot,{ }^{\prime}$ defined on it; and finally to the model in the semantic sense, which makes each sentence of $\mathrm{P}$ either true or false. As for the homomorphic images that we investigate, we shall consider only those induced by a principal ideal (nearly), determined

\footnotetext{
${ }^{3}$ Thanks to Dr. Gordon Monro for having suggested in conversation several years ago that this fact might prove useful from a Relevant viewpoint.
}

Australasian Journal of Logic (18:5) 2021, Article no. 7 
by a particular element $n$ of $\mathbf{M}$.

Specifically, given $n$ in $\mathbf{M}$, we define the structure $\mathbf{M}_{n}$ as follows: we define a relation $\equiv$ on $\mathbf{M}$ on the rubric $a \equiv b$ iff, for some $x$ in $\mathbf{M}$, either $a+x \cdot n=b$ or $a=b+x \cdot n$. It is elementary that $\equiv$ is a congruence on $\mathbf{M}$ with respect to the chose operations (using fundamental properties of $+, \cdot, 0$, ' and the fact that $\mathbf{M}$ is a model of $\mathrm{P}$ ), whence we shall say that two elements $a, b$ of $\mathbf{M}$ are congruent $\bmod n$ provided that $a \equiv b$. We may now pass, in the usual algebraic way, to congruence classes, forming the structure $\mathbf{M}_{n}$ of integers $\bmod n$. Since $\equiv$ is a congruence, the elements 0 and the operations $+, \cdot,{ }^{\prime}$ are well-defined on $\mathbf{M}_{n}$. And $\mathbf{M}_{n}$ is a natural homomorphic image of $\mathbf{M}$, on the morphism $h$ which takes each element $a$ of $\mathbf{M}$ to the set of elements congruent to it. We shall normally refer to the elements of the quotient algebra by their representatives, noting that for each congruence class there is exactly one member $b$ of $\mathbb{N}$ such that $b<n$ in $\mathbf{M}$ and which belongs to this class. (There cannot be two such representatives, since if $a$ and $b$ are congruent they must differ by a multiple of $n$, whence one of them will be no less than $n$. And, by the least number principle, the set of elements congruent to each $b$ must have a least member $a$, which will serve as a representative; for, if $a$ were not less than $n$, we could subtract $n$ and get a smaller representative. As usual, the "kernel" of the homomorphism-the set of elements congruent to 0 - consists exactly of the "integers" divisible by $n$.)

There are a couple of more or less silly choices of $n$, though we permit them for completeness. (Though, should we be careless in our statement of some theorem, we trust the reader to exclude the silly choices.) Setting $1=0^{\prime}$ as usual, we note that $\mathbf{M}_{1}$ is the trivial 1-element algebra. And $\mathbf{M}_{0}$, which identifies $a$ and $b$ just in case they differ by a multiple of 0 -i.e., not at all - is, for all practical purposes, just $\mathbf{M}$ itself. Except in this last case, $\mathbf{M}_{n}$ is a ring, even though $\mathbf{M}$ is not. Indeed, $\mathbf{M}_{n}$ is a commutative ring with unit. For its non-zero elements certainly form a commutative monoid under multiplication, with identity 1 . Equally certainly, $\mathbf{M}_{n}$ is an additive commutative monoid, with identity 0 . (In the other silly case $\mathbf{M}_{1}$, we have $0=1$.) Moreover, · distributes over + . So we need only show that there exists an additive inverse $-b$ for each $b$ in $\mathbf{M}_{n}$. Since the system $\mathbf{P}$ compels each non-zero element to have a predecessor, $n$ in particular has a predecessor (if $n \neq 0$ ), which we might as well call -1 . It is then apparent that $-1 \cdot b$ will have the right group-theoretic properties to be the inverse $-b$ of $b$ for each $b$ in $\mathbf{M}_{n}$.

Australasian Journal of Logic (18:5) 2021, Article no. 7 
The reader who was going to accept our invitation to skip the hard stuff but who decided to read on a bit must, by now, be feeling pretty superior. It has hit nothing yet that it could not easily have looked up or worked out in its CPU, in nanoseconds. Meanwhile, let us spell out what $\mathbf{M}_{n}$ does, as a semantic structure. (When we are thinking of it, we may refer to it as $\mathbf{M}_{n}^{2}$, to indicate that it has $\mathbf{2}=\{\mathrm{T}, \mathrm{F}\}$ as its set of truth-values.) Let $\mathscr{L}^{\sharp}$ be our arithmetical first-order language, which we think of as extended to a language $\mathscr{L}_{n}^{\sharp}$ by adjoining each member of $\mathbf{M}$ as a new individual constant (to be used as its own name). There is a natural interpretation function. $I_{n}^{2}$ associated with $\mathbf{M}_{n}^{2}$, whose arguments are all the closed terms and formulas of $\mathscr{L}_{n}^{\sharp}$ (and a fortiori of $\mathscr{L}^{\sharp}$ ), with values in $\mathbf{M}_{n}$ for closed terms and in $\{\mathrm{T}, \mathrm{F}\}$ for closed formulas, constructed on the usual recursive specifications. We may extend $I_{n}^{2}$ to all formulas, if we wish, by letting its value on an open formula be that of any universal closure of this formula. And $\mathbf{M}_{n}^{2}$ is then a model of any formula $A$ such that $I_{n}^{2}(A)=\mathrm{T}$; it is a model of a set of formulas iff it is a model of each formula in $S$. (Similar definitional remarks apply of course to $\mathbf{M}$ itself, whose associated interpretation function and extended language we shall call $I_{\mathbf{M}}^{2}$ and $\mathscr{L}_{\mathbf{M}}^{\sharp}$ respectively.)

We chose $\mathbf{M}$ as a model of $\mathbf{P}$. And $\mathbf{M}_{n}$, we know, is not a model of $\mathbf{P}$. But it is not far from one. Indeed, let $\mathrm{P}^{+}$be the system formulated like $\mathrm{P}$, but without the axiom $\forall x \sim\left(x^{\prime}=0\right)$ forbidding 0 from being a successor. Then

Observation 1. $\mathbf{M}_{n}^{2}$ is a model of $\mathrm{P}^{+}$.

We are indebted to Paris and Wilkie for pointing out in conversation that the observation is obvious. Indeed, that most of the first-order Peano axioms hold in $\mathbf{M}_{n}^{2}$ is immediate from the fact that it is a homomorphic image of $\mathbf{M}$. The only tricky one is mathematical induction. But this is not tricky either when we realize that congruence $\bmod n$ is already definable in the language $\mathscr{L}_{n}^{\sharp}$ itself, by

$$
D \equiv . \quad t \equiv u=_{\mathrm{DF}} \exists x(t=u+x \cdot n \vee t+x \cdot n=u)
$$

taken schematically for all terms $t$ and $u$. (Evidently we could define congruence mod an arbitrary term $w$ by putting $w$ for $n$ in the definiens. But since it is congruence mod this particular $n$ that we have most in mind, we shall stick to the special case where $n$ is a constant of our extended language.) Having fixed $n$ and used it to define $\equiv$ contextually, let $A$ be any formula of $\mathscr{L}_{n}^{\sharp}$ and let $A_{\equiv}$ be the result of systematically replacing all occurrences of 
"=" in $A$ (in primitive notation) with the defined "三." It is quickly observed that $A$ is valid in $\mathbf{M}_{n}^{2}$ iff $A_{\equiv}$ is valid in $\mathbf{M}$, in the usual classical sense. In particular, if $A$ is an instance of the scheme of mathematical induction, $A_{\equiv}$ is also an instance of mathematical induction. This is valid in $\mathbf{M}$, because it is a model of $\mathrm{P}$. Accordingly, $A$ is valid in $\mathbf{M}_{n}^{2}$, by the correspondence just noted. Everything else being straightforward, it is then obvious that $\mathbf{M}_{n}^{2}$ is a model of $\mathrm{P}^{+}$. This is the content of our observation, ending our sketch of its verification.

We now use the following recipe to turn a "mod" model $\mathbf{M}_{n}^{2}$ of $\mathrm{P}^{+}$into a "relevant" model $\mathrm{M}^{6}$ of all of $\mathrm{R}^{\sharp}$ of all of $\mathrm{R}^{\sharp}$. (Basically, it is an application of the technique used in [14] to show $R^{\sharp}$ a conservative extension of its negationfree mate $R_{+}^{\sharp}$.) First of all, consider the following Hasse diagram, of a lattice we henceforth call just 6 .

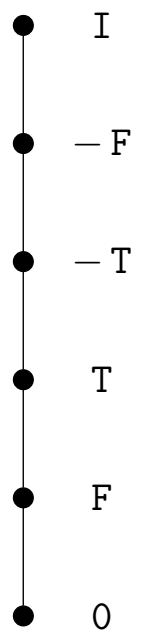

While our nomenclature for $\mathbf{6}$ differs from our usual policy (we generally reserve "F" for the bottom element and "T" for the top element of a DeMorgan monoid, as $\mathbf{6}$ will turn out to be), and have labelled things as we have to to make classical connections. As usual, \& will be interpreted as lattice meet, and $\vee$ as lattice join (in this case, just min and max in the displayed order). $\sim$ is order-reversing, subject to $\sim 0=\mathrm{I}, \sim \mathrm{F}=-\mathrm{F}, \sim \mathrm{T}=-\mathrm{T}$, etc. The truth-table for $\rightarrow$ is given as follows:

Australasian Journal of Logic (18:5) 2021, Article no. 7 


\begin{tabular}{r|rrrrrr}
$\rightarrow$ & 0 & $\mathrm{~F}$ & $\mathrm{~T}$ & $-\mathrm{T}$ & $-\mathrm{F}$ & $\mathrm{I}$ \\
\hline 0 & $\mathrm{I}$ & $\mathrm{I}$ & $\mathrm{I}$ & $\mathrm{I}$ & $\mathrm{I}$ & $\mathrm{I}$ \\
$\mathrm{F}$ & 0 & $\mathrm{~T}$ & $\mathrm{~T}$ & $-\mathrm{F}$ & $-\mathrm{F}$ & $\mathrm{I}$ \\
$\mathrm{T}$ & 0 & $\mathrm{~F}$ & $\mathrm{~T}$ & $-\mathrm{T}$ & $-\mathrm{F}$ & $\mathrm{I}$ \\
$-\mathrm{T}$ & 0 & 0 & 0 & $\mathrm{~T}$ & $\mathrm{~T}$ & $\mathrm{I}$ \\
$-\mathrm{F}$ & 0 & 0 & 0 & $\mathrm{~F}$ & $\mathrm{~T}$ & $\mathrm{I}$ \\
$\mathrm{I}$ & 0 & 0 & 0 & 0 & 0 & $\mathrm{I}$
\end{tabular}

What 6 is, really, is the result of taking truth-tables (viewed as a Dunn monoid) into a DeMorgan monoid, conservatively adding "DeMorgan negation" on the plan first set out in [9]. To make that point, we are using $\mathrm{F}$ and $\mathrm{T}$ to stand for (classical) false and true. (But $\mathrm{T}$ may also be identified with the intuitive "least truth" $\mathbf{t}$ required by DeMorgan monoid theory. But its mate, the "greatest falsehood" $\mathbf{f}$, is in fact $-T$. As "designated elements" of 6, count everything in the principal filter determined by $\mathrm{T}$ : i.e., all of $\mathrm{T},-\mathrm{T}$, $-F$, and I.)

It is readily verified that 6 satisfied the postulates on a DeMorgan monoid laid down by Dunn in [5]. (The lazy need merely note that it turns up among the structures generated by the program TOPSY - so named by Slaney because it "just growed" - explained in [18].) Since DeMorgan monoids stand to $\mathrm{R}$ as Heyting lattices stand to intuitionism, this means that $\mathbf{6}$ will look after the relevant verification of all quantifier-free logical postulates; and, being finite (and hence a complete lattice) it will also look after the verification of relevant logical postulates containing quantifiers, with $\forall$ interpreted as a generalized meet and $\exists$ as a generalized join (which, in a simply ordered case like $\mathbf{6}$, means just that $\forall x A x$ will get the "falsest" value of any of its instances $A t$, while $\exists x A x$ will get the "truest.")

What is nicest about $\mathbf{6}$, for our immediate purposes, is that it offers a convenient way to turn classical theories, and their accompanying models, into relevant ones, while still preserving relevant distinctions. Many of these distinctions have to do with negation. The DeMorgan $\sim A$ denies $A$. But it does not, like its Boolean cousin, confuse the denial of $A$ with the assertion that $A$ implies anything whatsoever. (Boolean negation is nonetheless an interesting and valuable connective of relevant logics, unjustly pilloried by authors of whom we otherwise speak well. But there is not time to go into all that here.) Thus from one viewpoint - there are others, consistent with relevant insight - classical logic simply lacks negation. Or rather it confuses negation with $A \rightarrow \mathrm{F}$, where the distinguishing feature of $\mathrm{F}$ is that it im- 
plies everything (classically) in sight. As is well-known, $0=1$ will do as such an F arithmetically. So we might rethink classical theories as positive theories, whose logical particles are just \&, $\vee, \rightarrow, \forall, \exists$, and which introduce (what passes for) negation via some sentence silly enough to imply absolutely everything.

While we are not necessarily recommending this view of classical negation, let's try it out. We would then view classical logic as a positive logic, consisting of all the classical tautologies in the positive particles just listed. A classical theory would then be any theory $T$ which contained all those tautologies and which was closed under $\rightarrow E$. If a classical theory does contain an $\mathrm{F}$ that implies everything, according to this theory, then it is moreover classical in the usual sense, defining (classical) not- $A$ as $A \rightarrow \mathrm{F}$. Let us call such a theory a classical F-theory. Provided F is not itself a theorem of the theory (which would rather spoil things), any classical F-theory will have the usual models. It is time for a theorem.

Model Transformation Theorem. Let $\mathbf{M}^{2}$ be any model of a classical F-theory, in the usual sense. We may extend $\mathbf{M}^{2}$ to a model $\mathbf{M}^{6}$, in the DeMorgan monoid $\mathbf{6}$, with the following properties.

(1) All theorems of the first-order relevant logic $\mathrm{RQ}$ are valid in $\mathbf{M}^{6}$

(2) The class of valid sentences is closed under the rule $\rightarrow E$ (modus ponens for $\rightarrow$ ) and the rule \&I of $\mathrm{RQ}$

(3) Every sentence true in $\mathbf{M}^{2}$ takes the value $\mathbf{T}$ in $\mathbf{M}^{6}$ (and is hence a "good guy," by the lights of $\mathbf{M}^{6}$ )

(4) Every sentence false in $\mathbf{M}^{2}$ takes the value $\mathbf{F}$ in $\mathbf{M}^{6}$ (and is hence a "bad guy," by those same lights)

(5) Let $A$ be any positive sentence; then $\sim A$ takes a designated value in $\mathbf{M}^{6}$

(Accordingly, adding any class of such sentences as new relevant axioms produces a conservative extension of the classical theory corresponding to the truths in $\mathbf{M}^{2}$, and a fortiori produces a conservative extension of the original classical F-theory, understood now as a relevant theory.)

Explanation 1. Before proving this theorem, we wish to remind the reader what it means. We are thinking of our F-theory as lacking $\sim$ in its formation apparatus, while containing all positive particles, including $\rightarrow$, which the 
theory in question treats as material implication. From this viewpoint, extending $\mathbf{M}^{2}$ to $\mathbf{M}^{6}$ is introducing negation, taken as a new primitive particle $\sim$, and subject to all usual negation laws (double negation, excluded middle, non-contradiction, etc.) except paradoxical ones. It then turns out, in line with previous results, that we can be quite free about adding axioms of the form $\sim A$, or their relevant equivalents, when $A$ is in the old, positive vocabulary. (These things are essentially known, from [11]. What is striking is that, if we start from a classical theory, they can be put very simply.)

Proof. On well-known syntactical maneuvers, we may assume that only sentences (i.e., formulas without free variables) enter into our stipulations of logics and theories. We also assume, in specifying the modelling conditions, that the language of a theory is enriched to include names for all elements of the model, as above. Assuming that $\mathbf{M}^{2}$ has been given, with an associated interpretation function $I^{2}$ defined on all closed terms and sentences of our (positive) enriched language with values in $\{F, T\}$ for sentences, we characterize $\mathbf{M}^{6}$ and its associated interpretation function $I^{6}$ as follows.

(i) The domain $\mathbf{D}$ of objects of $\mathbf{M}^{6}$ shall coincide with the objects of $\mathbf{M}^{2}$, and $I^{6}$ shall coincide with $I^{2}$ on all closed terms.

(ii) The domain of truth-values of $\mathbf{M}^{6}$ shall be the lattice $\mathbf{6}$ displayed above.

(iii) $I^{2}$ and $I^{6}$ shall coincide on atomic formulas, identifying classical T, F with the T, F of 6 .

(iv) On propositional connectives, $I^{6}$ shall be homomorphic; i.e., $I^{6}(\sim A)=\sim I^{6}(A), I^{6}(A \rightarrow B)=I^{6}(A) \rightarrow I^{6}(B)$, etc.

(v) $\quad I^{6}(\forall x A x)=I^{6}(A t)$, where this value is least under $I^{6}$ for any sentence of the form $A t$, where $t$ ranges over the closed terms of the extended language.

(vi) $\quad I^{6}(\exists x A x)=I^{6}(A t)$, for the greatest such value of an $A t$ under $I^{6}$.

(vii) A sentence is verified in $\mathbf{M}^{6}$ iff it takes a value $\geq \mathrm{T}$ under $I^{6}$ in the lattice ordering of $\mathbf{6}$. I.e., $A$ is verified if $I^{6}(A) \in\{\mathrm{T},-\mathrm{T},-\mathrm{F}, \mathrm{I}\}$.)

While that was somewhat long-winded, how the specification operates is clear. $I^{6}$ extends $I^{2}$ by looking after formulas not in the original positive vocabulary; in particular, those containing DeMorgan $\sim \mathbf{M}^{6}$ has extra truth-values to look after that extension. The particular assertions of the 
theorem are now straightforward. Because $\mathbf{6}$ is a finite DeMorgan monoid, any interpretation $I$ therein is going to verify first-order relevant axioms and rules; this disposes of (1) and (2). Since $I^{2}$ and $I^{6}$ coincide on all positive sentences, (3) and (4) are also immediate. As for (5), a positive sentence must take one of the values $\mathrm{T}, \mathrm{F}$; so its negation must take one of $-\mathrm{T},-\mathrm{F}$, either of which suffices to verify it in $\mathbf{M}^{6}$. Moreover, since the class of sentences verified in $\mathbf{M}^{6}$ contains all relevant tautologies, all sentences in our F-theory, all negations of positive sentences and is closed under relevant rules, it is evidently a regular relevant theory which extends the F-theory we started with; but, since every non-theorem of this theory is refutable in some $\mathbf{M}^{2}$, and hence in a corresponding $\mathbf{M}^{6}$, any relevant denials of positive formulas may be added conservatively, in accordance with our parenthetical remarks. This completes the proof of the model transformation theorem.

Having the model transformation theorem in hand, we can now return to our previous considerations. Here is a corollary to look after them.

Corollary 1. Let $\mathbf{M}$ be any model of classical first-order Peano arithmetic $\mathbf{P}$. Let $n \neq 0$ be an element of $\mathbf{M}$, and let $\mathbf{M}_{n}^{2}$ be the classical model of $\mathrm{P}^{+}$"modulo n," with interpretation function $I_{n}^{2}$. Then $\mathbf{M}_{n}^{6}$, univocally determined by $\mathbf{M}_{n}^{2}$ by the recipe of the theorem, is a model of $\mathbf{R}^{\sharp}$, which verifies exactly the same positive sentences that $\mathbf{M}_{n}^{2}$ makes classically true; moreover, the "integers" of $\mathbf{M}_{n}^{6}$ are just those of $\mathbf{M}_{n}^{2}$.

Proof. Everything follows immediately from the theorem, except the statement that $\mathbf{M}_{n}^{6}$ is a model of $\mathrm{R}^{\sharp}$. Remember that, classically speaking, $\mathbf{M}_{n}^{2}$ failed to verify the postulate which says that 0 is a non-successor. But that means, under our "positive translation" of $\mathrm{P}^{+}, \exists x\left(x^{\prime}=0\right) \rightarrow 0=1$ (near enough). Clearly this deserves to be a non-theorem of $\mathrm{P}^{+}$, since it is truth-functionally refuted mod 2 It is a different matter, relevantly, to assert $\forall x\left(\sim x^{\prime}=0\right)$ (which, of course, is completely equivalent to $\sim \exists\left(x^{\prime}=0\right)$ ). As a Peano postulate, and hence as an axiom of $R^{\sharp}$, it has to be verified in any model of $R^{\sharp}$. This may surprise the reade who skimmed our earlier remarks, but who has remembered that, in $\mathbf{M}_{n}^{2}, 0$ is a successor; specifically, it is the successor of what we playfully called -1 above. But recall Dunn's interpretation of our policy in such matters; we are dealing with a "confused" homomorphic picture of an $\mathbf{M}$ in which 0 was not a successor. This suggests, as before, that both $\exists x\left(x^{\prime}=0\right)$ and its negation should be verified in $\mathbf{M}_{n}^{6}$. And this is in fact what happens; since $-1^{\prime}=0$ according to $\mathbf{M}_{n}^{6}$, 
$\left(\exists x\left(x^{\prime}=0\right)\right)=\mathrm{T}$; but then its negation takes the value $-\mathrm{T}$, also a "good guy," (Indeed, according to our theorem, we could add the negation of any positive formula, or a relevant equivalent, conservatively as a new axiom.) So the non-successor postulate, as an axiom of $\mathrm{R}^{\sharp}$, is verified after all.

Otherwise, there is almost nothing to the verification $\mathbf{M}_{n}^{6}$ is a model of $\mathbf{R}^{\sharp}$. The logical axioms and rules, and all positive proper axioms, are looked after by the theorem. This covers everything but mathematical induction. For this principle is schematic, of the form $A 0 \& \forall x\left(A x \rightarrow A x^{\prime}\right) \rightarrow \forall x A x$. Evidently, if $A x$ is a positive formula, the corresponding instance of mathematical induction will be verified in accordance with the model transformation theorem in $\mathbf{M}_{n}^{6}$, given that we have already observed that it is true in $\mathbf{M}_{n}^{2}$. But $A x$ may contain DeMorgan negation, which is not covered directly by the theorem. So it is necessary, as in [14], to dig a little deeper. We observe first that, where $A x$ is any formula in which at most $x$ occurs free, $\mathbf{M}_{n}^{6}$ constrains the interpretative possibilities as follows:

(a) For all closed terms $t, I_{n}^{6}(A t) \in\{\mathrm{T}, \mathrm{F}\}$. Or

(b) for all closed terms $t, I_{n}^{6}(A t) \in\{-\mathrm{T},-\mathrm{F}\}$. Or

(c) for all closed terms $t, I_{n}^{6}(A t)=0$. Or

(d) for all closed terms $t, I_{n}^{6}(A t)=\mathrm{I}$.

Proof is by induction on the complexity of $A x$. (a) holds if $A x$ is atomic. The other cases are settled by straightforward inductive argument, which we leave to the reader. It is then evident that, in cases (c) and (d), the induction axiom must take the value I, verifying it. We next wish to show that, in case (a), we have $A x$ equivalent in the model $\mathbf{M}_{n}^{6}$ to a positive formula, in the sense that there exists a negation-free formula $B x$ such that $\forall x(A x \leftrightarrow B x)$ is valid in the model; and that, in case (b), we have $A x$ equivalent in the model to the negation of a positive formula. Again we argue by induction on the length of $A x$, the atomic case being immediate. Again leaving details to the reader, this settles case (a), replacing equivalents and noting once more that induction holds for $A x$ positive, by construction. But it also settles case (b); for, in this case, $\forall x A x$ must be one of $-\mathrm{T},-\mathrm{F}$ on interpretation; while $\forall x\left(A x \rightarrow A x^{\prime}\right)$ must be one of $\mathrm{T}, \mathrm{F}$, which will force the conjunction in the antecedent of the induction scheme to precede the value given to the consequent in the ordering of $\mathbf{6}$ under interpretation. This completes the verification of the induction postulate in $\mathbf{M}_{n}^{6}$, and with it the proof of the 
corollary from the preceding theorem.

After all this, we may exhibit a model of $\mathrm{R}^{\sharp}$ with alien intruders aplenty.

Alien Intruder Theorem. Every rational number is a non-negative integer. That is, there is a model $\mathbf{M}$ of $\mathrm{R}^{\sharp}$ such that the following obtain, in a straightforward sense.

(a) Every rational number is an element of $\mathbf{M}$.

(b) The ordinary laws of rational arithmetic hold, for addition, multiplication, subtraction, division.

(c) The Peano postulates are satisfied by $\mathbf{M}$, including mathematical induction.

Proof. Add a new constant $n$ to the vocabulary of $\mathrm{P}$. Define $\equiv$ as above as congruence $\bmod n$. Form an extension $T$ of $\mathrm{P}$ by adding as new axioms $\forall x(x \equiv 0 \vee \exists y(x \cdot y \equiv 1))$; and, for each numeral $m$ corresponding to a "standard" natural number, $\sim m \equiv n$. $T$ must be a consistent theory. For, if it is inconsistent, some finite conjunction of these added axioms must be inconsistent; however, there is then a standard number $p$ such that (i) $p$ is prime and (ii) all numbers named in the inconsistent finite conjunction of added axioms are less than $p$. But, interpreting $n$ as $p$, we have a model for the finite subset of axioms alleged to be inconsistent (for no number smaller than $p$ is congruent to $p \bmod p$; while since the integers $\bmod$ a prime number constitute a field, the axiom asserting the existence of a multiplicative inverse for each element not congruent to $0 \bmod p$ will also hold.) So, by compactness, $T$ has a model, which we may call $\mathbf{M}^{*}$. Forming congruence classes $\bmod n$, we get a model $\mathbf{M}_{n}^{*}$ of $\mathrm{P}^{+}$, which is then transformed into a model $\mathbf{M}_{n}^{6}$ of $\mathrm{R}^{\sharp}$ in accordance with the preceding corollary. This is our desired M, in which the Peano postulates hold (in their first-order relevant version) by the corollary. We must now show that all rational numbers are admitted by $\mathbf{M}$.

In the first place, since no standard natural number was congruent to $n$ in $\mathbf{M}^{*}$, every standard number is an element of the quotient algebra $\mathbf{M}_{n}^{*}$ (and hence of $\mathbf{M}$, which has the same objects in its domain). Using -1 as before to denote the element whose successor is 0 , for each standard positive integer $m$ in $\mathbf{M}$ there will be a corresponding standard negative integer $-m=$ $(-1) \cdot m$; moreover, since $\mathbf{M}$ is in the natural way a ring, these standard 
integers constitute a sub-ring of $\mathbf{M}$, as expected. But, for each non-zero integer $m$, our added postulate guarantees a reciprocal $1 / m$. (The notation is justified, since multiplicative inverses must be unique. For suppose, to contrary, that there are distinct $i$ and $j$ such that $m \cdot i=m \cdot j=1$. But then $j=1 \cdot j=i \cdot m \cdot j=i \cdot 1=i$, whence $i$ and $j$ are not distinct after all.) We may then form the arbitrary rational fraction $k / m$ as $k \cdot(1 / m)$, for $m \neq 0$, in $\mathbf{M}$. For $k, m$ rational standard integers, these fractions may be reduced to lowest terms in the usual way; the usual laws of rational arithmetic will thereupon hold, ending the proof of the theorem.

All of this is, to say the least, most startling and wonderful. Bereft of the paradoxical properties of classical negation, it is possible to satisfy the Peano postulates in a domain (indeed, in many domains, since every non-standard model $\mathbf{M}^{*}$ of $\mathrm{P}$ may be transformed into a model $\mathbf{M}$ of $\mathrm{R}^{\sharp}$ in this fashion) that contains all the rationals, among the alien intruders. The price of this, to be sure, is inconsistency; in the model-theoretic sense, because $\mathbf{M}$ verifies both $A$ and $\sim A$ for some choices of $A$; and in the syntactic sense, taking the class of sentences verified in $\mathbf{M}$ as a relevant theory that extends $R^{\sharp}$ (as it is) but which contains explicit contradictions of the form $A \& \sim A$. For those addicted to the "foolish hobgoblin of little minds," there is little that we can say at this point; for those who take consistency to be the hallmark and only criterion for mathematical existence, there is even less. But, to be honest, there was never much to be said for this criterion. In whatever shadowy sense mathematical entities may be said to exist, their interest lies in the beauty and richness of the structures to which they give rise, and to the possibility of applying these structures to the real as opposed to the mathematical world. No one will deny that rational numbers are applicable to the world; if, in applying them, we unmask them as just another sort of integer, we can view that as just another beautiful and interesting fact about them. And the trouble with inconsistency, as fatal to mathematical existence, lies not with the inconsistent as such; but in the fact that, if negation is allowed its horrendous paradoxical classical properties, the presence of a single inconsistency calls the mathematical game off, allowing everything to be proved. If the game can still go on-indeed, even perhaps become more interesting - if we adopt relevant and not merely truth-functional canons of inference, then there is no reason in principle not to do so. As a recipe for reconstructing mathematical reason, there always was a good deal wrong with classical logic - if only because intuitive reason is

Australasian Journal of Logic (18:5) 2021, Article no. 7 
subject to those relevant constraints that its truth-functional regimentation ignores. If we attend to those constraints in our reconstruction of logic, and do not elevate ignoring them to the level of unreasoned dogma, there are mathematical worlds undreamed of yet to conquer.

Meanwhile, it is interesting to see how far we can go along the lines just laid out. Can we, for example, construct a model of $\mathrm{R}^{\sharp}$ on the above plan whose elements are exactly the standard set $\mathbb{Q}$ of rational numbers? The answer is "No." For, among the theorems of $\mathrm{P}$, there are assertions like, "Every natural number is the sum of four squares." These assertions are preserved on the $\mathbf{M}_{n}$ style passages to homomorphic images, and on to the resulting "inconsistent" models of $R^{\sharp}$. In these homomorphic images, -1 is a number. Accordingly, -1 is the sum of four squares. Evidently, when all rational numbers are elements of our model, -1 cannot be the sum of four squares of rationals. So any model of this sort which includes the rationals must include other elements, not to be identified with any rational number. (As, on a little reflection about the subject, is perfectly clear anyway.)

But the method which produced the Alien Intruder Theorem will go a lot further. Perhaps, for example, one would like to have the imaginary number $i$ among the integers, without identifying it with any standard integer (recalling, e.g., that $4^{2}=-1 \bmod 17$, whence $4=i$ from that viewpoint; to be sure, so is 13, which is a little disturbing). Well, we can pull the same trick. Add $\exists x\left(x^{2}+1 \equiv 0\right)$, while denying for each numeral $m$ that $m^{2}+1 \equiv 0$. The result, by compactness, is a consistent extension of $\mathrm{P}$, which must have a model containing an element $i$ such that $i^{2}=-1$, where $i$ is distinct from all rational numbers when we collapse mod the appropriate congruence. Question: can we view all real numbers as integers? Answer: we don't know.

But, the reader may protest, what you have done is extremely silly. Above all, the natural numbers are characterized by induction. How can, say, 3/2 count seriously as an admissible alien intruder? Could anybody give as a serious reason for $3 / 2$ possessing a certain property (i) that having that property $P$ is preserved under adding 1's and (ii) 0 has $P$ ? We can, to be sure, conclude on that basis that 0 has $P, 1$ has $P, 2$ has $P$, etc. But, in the process, we seem to have skipped $3 / 2$ on our way from 1 to 2 . Would it not be magical if $3 / 2$ had the property $P$ as well?

We should like to respond, first, that it is magical that induction should hold anywhere except in the standard model. We do not get to ordinary nonstandard integers either from 0 by adding 1's; yet, in any non-standard model 
of $\mathrm{P}$, these greatly outnumber the standard integers. But, second, the idea actually sounds less silly with respect to the rational numbers - which after all are elements of a well-known and rather intuitive structure - than it does of the usual "non-standard integers." If our arithmetical intuitions start with natural numbers, on what basis do we intersperse or tack on extra elements to a well-known number sequence? There are, after all, all kinds of structures in which the natural numbers can be embedded. Some of these embeddings have themselves come to be thought "natural" - into the integers, the rationals, the reals; and, more recently, into the ordinals and cardinals. Non-standard models of the usual sort, from this viewpoint, were just more of the same.

All of these embeddings are characterized not merely by the fact that the natural numbers can be located in the resulting structure, but also by the fact that certain laws governing the natural numbers are preserved (and extended) as we enrich the number system. But, from this viewpoint, we must ask, "Which laws are to be preserved? And why do we believe those laws in the first place?" This leads us not merely to mathematical induction, but to something resembling scientific induction. A facetious answer to the question, "Why should $3 / 2$ have property $P$ if 0 has property $P$ and successors of $P$ have $P$ ?" might be "Well, if all those guys have $P$, any scientist would believe that $3 / 2$ has $P$ also, in the absence of specific reason to believe the contrary!"

Behind this facetious answer there is a point. What sorts of laws do we have an interest in preserving? Typically, ones like " $x+y=y+x$," " $x \cdot(y+z)=(x \cdot y)+(x \cdot z)$." (To be sure, we aren't forced to preserve them; e.g., on generalization to ordinals, commutativity of + fails; but such failures tend to result because there is something else that we have an interest in preserving - in the case of ordinals, that every non-empty set of them has a least element.) But why should we have believed that addition was commutative in the first place? Presumptively, mathematical induction gives a reason. And if we then wish to hold " $(3 / 2)+y=y+(3 / 2)$," is there nothing to the thought that, since mathematical induction has conferred this property on the natural numbers, we want it to hold of the rationals also? And since we have long thought of integers as special sorts of rational numbers, perhaps it is time to return the favour; alien intruders they may be; but, by our theorem, rational numbers are conversely special sorts of integers.

It will not do, to be sure, to push these sorts of speculation too far. But they do raise food for thought, as well as interesting directions for research. Another view of a mathematical structure never hurts. For, if all else fails, 
it might help us to prove a theorem that has hitherto escaped us. Or give us some further insight into what we wish to prove. And why.

\section{Acknowledgments}

Thanks to members of the Automated Reasoning Project, A.N.U. for discussion of this paper. And to members of the Universities of Sydney, Melbourne, and Newcastle for further discussion.

\section{References}

[1] A. R. Anderson and N. D. Belnap. Entailment, volume I. Princeton University Press, Princeton, 1975.

[2] N. D. Belnap. Intensional models for first degree formulas. Journal of Symbolic Logic, 32(1):1-22, 1967.

[3] J. P. Burgess. Commonsense and relevance. Notre Dame Journal of Formal Logic, 24(1):41-53, 1983.

[4] C. C. Chang and H. J. Keisler. Model Theory. North-Holland, Amsterdam, 1973.

[5] J. M. Dunn. The Algebra of Intensional Logics. PhD thesis, University of Pittsburgh, 1966.

[6] J. M. Dunn. A theorem in 3 valued model theory with connections to number theory, type theory, and relevant logic. Studia Logica, 38(2):149$169,1979$.

[7] K. Gödel. Über formal unentscheidbare Sätze der Principia Mathematica und verwandter Systeme I. Monatshefte für Mathematik und Physik, $38: 173-198,1931$.

[8] H. Grassmann. Lehrbuch der Arithmetik. Verlag von T. C. F . Enslin, Berlin, 1861.

[9] R. K. Meyer. On conserving positive logics. Notre Dame Journal of Formal Logic, 14(2):224-236, 1973.

Australasian Journal of Logic (18:5) 2021, Article no. 7 
[10] R. K. Meyer. The consistency of arithmetic. Unpublished monograph, 1976.

[11] R. K. Meyer. Negation disarmed. Notre Dame Journal of Formal Logic, 17(2):184-190, 1976.

[12] R. K. Meyer. Relevant arithmetic. Bulletin of the Section of Logic, 5:133-137, 1976.

[13] R. K. Meyer and C. Mortensen. Inconsistent models for relevant arithmetics. Journal of Symbolic Logic, 49(3):917-929, 1984.

[14] R. K. Meyer and I. Urbas. Conservative extensions in relevant arithmetic. Zeitschrift für mathematische Logic und Grundlagen der Mathematik, 32:45-50, 1986.

[15] C. Mortensen. Inconsistent nonstandard arithmetic. Journal of Symbolic Logic, 52(2):512-518, 1987.

[16] C. Mortensen. Inconsistent number systems. Notre Dame Journal of Formal Logic, 29(1):45-60, 1988.

[17] T. Skolem. Über einige grundlagenfragen den mathematik. In Skifter Vitenskabsakademiet $i$ Oslo, volume I, pages 1-49. 1929.

[18] J. K. Slaney. Computers and Relevant Logic: A Project in Computing Matrix Model Structures for Propositional Logics. PhD thesis, Australian National University, 1980.

[19] J. van Heijenoort. From Frege to Gödel: A Source Book in Mathematical Logic, 1879-1931. Harvard University Press, Cambridge, MA, 1967.

[20] H. Wang. The axiomatization of arithmetic. Journal of Symbolic Logic, $22(2): 145-157,1975$.

Australasian Journal of Logic (18:5) 2021, Article no. 7 